\title{
Assessment of the Successful External Cephalic Version Prognostic Parameters Effect on Final Mode of Delivery
}

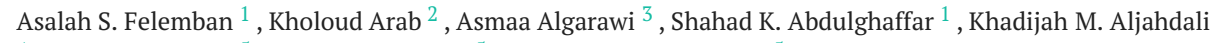
4 , Malak A. Alotaifi ${ }^{5}$, Sumayya A. Bafail ${ }^{5}$, Tasneem M. Bakhudayd ${ }^{5}$

1. Medicine, King Abdulaziz University Hospital, Jeddah, SAU 2. Obstetrics and Gynecology, King Abdulaziz University Hospital, Jeddah, SAU 3. Faculty of Medicine, King Abdulaziz University Hospital, Jeddah, SAU 4. Pediatrics, King Abdulaziz University Hospital, Jeddah, SAU 5. Faculty of Medicine, King Abdulaziz University, Jeddah, SAU

Corresponding author: Asalah S. Felemban, asalah.felemban@gmail.com

\section{Abstract}

\section{Aim}

This study aims to evaluate the prognostic parameters of successful approach for an external cephalic version (ECV) procedure by considering the vaginal delivery as the optimal mode of delivery.

\section{Methodology}

A retrospective cohort study was done during June 2019 in the obstetrics and gynecology department at King Abdulaziz University Hospital. Data were collected between May 2009 and May 2019 and included all pregnant women who were candidates for the ECV. The primary objective was to assess the final mode of delivery in relation to the outcome of ECV followed by the secondary objective which was the prognostic parameters of the ECV procedure (body mass index, amniotic fluid index, parity, estimated fetal weight). Additional variables were maternal age, placental position and ethnicity.

\section{Results}

We have studied 86 pregnant women with ECV attempts the overall ECV success rate was for 46 women (59.7\%). For the final mode of delivery, after a successful ECV procedure, 40 women (87\%) whom had spontaneous vaginal delivery, in association to successful ECV, the prognostic parameters recorded the highest success rate were multiparous 35 (76.1\%), body mass index between 25 and $29.9(53.1 \%)$, women older than 30 years old (60.9\%), gestational age between 37 to 39.6 weeks (56.5\%). Posterior placental location $55.6 \%$, estimated fetal weight more than 2500 (73.9\%).

\section{Conclusion}

Review began 07/01/2021 Review ended 07/26/2021 Published 07/26/2021

\section{○ Copyright 2021}

Felemban et al. This is an open access article distributed under the terms of the Creative Commons Attribution License CC-BY 4.0., which permits unrestricted use, distribution, and reproduction in any medium, provided the original author and source are credited.
Successful ECV cases have recorded a significant increase in the incidence of spontaneous vaginal delivery and the outcome of ECV which is affected by many prognostic parameters such as parity, maternal age, gestational age, body mass index, amniotic fluid index (AFI) and estimated fetal weight (EFW).

\section{Categories: Family/General Practice, Obstetrics/Gynecology}

Keywords: amniotic fluid index, external cephalic version, body mass index: bmi, ksa:kingdom of saudi arabia, estimated fetal weight, parity

\section{Introduction}

Breech presentation occurs in 3\% to $4 \%$ of all pregnant women at term [1-4]. The fatal outcome of breech deliveries is responsible for $5.8 \%$ of perinatal and neonatal mortality up to 28 days post-delivery among the term singleton deliveries in the Netherlands [5]. External cephalic version (ECV) has been proven to be effective in the reduction of the frequency of breech presentation after 36 or 37 weeks of gestation and consequently the number of cesarean deliveries for this condition [6,7] and is defined as the physical uterine maneuvering to change a breech positioned fetus to a vertex position by an expert obstetrician with ultrasound guidance [8]. The procedure is performed by applying pressure externally to the fetus through the gravid abdomen [7].

The success of ECV is extremely variable, ranging from $35 \%$ to $86 \%$ depending on different variables which affect the outcome of ECV procedure such as BMI, maternal age, amniotic fluid index, gestational age, parity, location of placenta, time in breech, presentation before the procedure and the ultrasonography $[2,4,6,8-11]$. According to a cohort study that was done they discovered these results: 2614 females (with average BMI $23.5 \mathrm{~kg} / \mathrm{m} 2$ ) with breech and no previous cesarean section had undergone ECV, and $49 \%$ were effective. ECV has been more effective in multiparas (64\%) than in nulliparas (40\%) [5]. 
According to another study that was attempted at Portland, USA they discovered that the incidence of effective ECV in each maternal parameter was considerably different and it showed that the greatest parameters of effective ECV were BMI and maternal height, as maternal height rises, the chances of an effective ECV rises, while the chance of an effective ECV declines as BMI rises [4]. Providing that, literature had documented that ECV is significantly contributing in decreasing the rate of cesarean sections (45\%, $65 \%, 55 \%-27 \%$ ), respectively $[2,3,12]$. Even though, $25 \%$ of women in the US and $50 \%$ in Canada who were carrying breech fetuses had undergone cesarean delivery while they were able to deliver vaginally $[2,3,12]$. While as, in Jeroen Bosch Hospital the vaginal delivery rate has increased from 43\% in 2013 to $71 \%$ in 2017 [11]. Few studies had efficiently assessed these parameters effectiveness in association to the final mode of delivery.

This study will evaluate the prognostic factors of successful approach for an ECV procedure by considering the vaginal delivery as the definitive mode of delivery and the optimal outcome.

\section{Materials And Methods}

We conducted a retrospective cohort study that was approved by the institutional review board (IRB), in June 2019 at King Abdulaziz University Hospital (KAUH) a tertiary center in Jeddah, the western region of Saudi Arabia, by using medical records (Phoenix) and archives.

From May 2009 until May 2019 under the department of obstetrics and gynecology, we included all pregnant women who were candidates for ECV, the eligibility criteria were pregnant women with a singleton fetus in breech position who were not having contraindications for ECV procedure. We excluded from our study women with multiple pregnancies or uterine anomalies, pregnancies complicated by placenta previa, premature ruptured membrane, antepartum hemorrhage and pre-eclampsia. The primary outcome was to assess the prognostic factors of successful approach for an ECV procedure by considering the vaginal delivery as the definitive mode of delivery and the optimal outcome. The secondary outcomes were prognostic parameters and its association with successful ECV in King Abdulaziz University Hospital.

Our primary predictors of interest were maternal body mass index (BMI), amniotic fluid index (AFI), parity, estimated fetal weight (EFW) and the outcome of ECV. Maternal BMI was calculated using the equation (BMI $=\mathrm{kg} / \mathrm{m}^{2}$ ) which was measured at 36 to 41 weeks of gestation; pregnant women were assigned to a BMI category according to standard WHO classification. Amniotic fluid index (AFI) was calculated by adding the anteroposterior diameters of the largest empty fluid pocket (no fetal parts or umbilical cord) in each quadrant. The AFI was considered normal between 5 and $25 \mathrm{~cm}$ while readings less than $5 \mathrm{~cm}$ were considered as oligohydramnios and more than $25 \mathrm{~cm}$ as polyhydramnios. Additional variables under investigation were maternal age, height, placental position and ethnicity.

All statistical analyses were performed using Excel 2019 and SPSS version 21 (IBM Corp., Armonk, NY). Univariate analysis was done by measuring the frequency and describing the data using measurements of central tendency and standard deviation. Bivariate chi-squared analysis was carried out on the prognostic parameters findings with the outcome of ECV procedure and its association with final mode of delivery whether it was vaginal or cesarean section. T-test was also used to calculate the statistical significance between the outcome of ECV and maternal age, estimated fetal weight and the pregnant women body mass index. P-value $<0.05$ was considered to indicate statistical significance. The prevalence was given in percentage with $95 \%$ confidence level.

\section{Results}

We have studied 86 pregnant women with ECV attempts that were made between May 2009 and May 2019. Of whom, 10 were rolled out as their files were inactive.

Accordingly, $77 \mathrm{ECV}$ attempts were analyzed. The primary outcome was to evaluate the final mode of delivery among pregnant women with successful ECV attempts. In which, the overall ECV success rate was 46 women (59.7\%) and the failure rate was 31 women (40.3\%).

For the final mode of delivery 50 women (64.9\%) had spontaneous vaginal delivery, 18 women (23.4\%) had elective cesarean delivery and nine women (11.7\%) had an emergency cesarean delivery as shown in Table 1 . 


\section{Cureus}

\begin{tabular}{|c|c|c|c|c|}
\hline Mode of delivery & SVD $64.9 \%(n=50)$ & \multicolumn{2}{|c|}{$\operatorname{ELCS} 23.4 \%(n=18)$} & EMCS $11.7 \%(n=9)$ \\
\hline Success $=59.7 \%(n=46)$ & $87 \%(n=40)$ & \multicolumn{2}{|c|}{$8.7 \%(n=4)$} & $4.3 \%(n=2)$ \\
\hline Failure $=40.3 \%(n=10)$ & $32 \%(n=10)$ & \multicolumn{2}{|c|}{$45.2 \%(n=14)$} & $22.6 \%(n=n)$ \\
\hline & SVD & ELCS & EMCS & Total \\
\hline Successful ECV & 40 & 4 & 2 & 46 \\
\hline Failure ECV & 10 & 14 & 7 & 31 \\
\hline Total & 50 & 18 & 9 & 77 \\
\hline
\end{tabular}

\section{TABLE 1: ECV outcome and the mode of delivery.}

ECV: external cephalic version; SVD: spontaneous vaginal delivery; ELCS: elective cesarian section; EMCS: emergency cesarian section.

Secondary outcome was to assess the effectiveness of the prognostic parameters with successful ECV procedures. For the gravidity status, there were 25 nulliparous (32.5\%) and 52 multiparous (67.5\%). Of these, 71 cases $(92.2 \%)$ admitted from antenatal ward and 6 (7.8\%) cases from the emergency department. The mean maternal age was $30.195(\mathrm{SD}=5.3362$ ) with a maximum age of 42 and a minimum of 20 , there was a statistical difference between the maternal age and the outcome of the ECV (P-value $=0.028$ ). The mean BMI of total ECV was 30.48 (SD 5.188) with a range that equals 23-44. The mean gestational age was 37.160 (SD 2.82), estimated fetal weight mean was $2686 \mathrm{~g}$ (SD 748.53). Ultrasonography exhibited multiple placental locations were 45 (58.4\%) posteriorly followed by 21 (27.3\%) anteriorly other locations accounted for $15 \%$ (anterior-fundal, posterior-fundal) and the data was not available for 2 (2.6\%). All women who underwent ECV had an appropriate amniotic fluid index.

Our data had documented 12 different nationalities. The percentage of Saudi women was $62.3 \%$, followed by Yemeni women with 13\%, Palestinian with 5.2\%, Sudanese with 3.9\%, Pakistanis, Indians, Filipinos and Bangladeshi with 2.6\%, Chadian, Burmese, Jordanian and Afghani with 1.3\%. On assessing the pregnant women condition there were chronic history of diabetes mellitus in three women (3.9\%), maternal hypertension in one woman (1.3\%), gestational diabetes in three (3.9\%) women and none of them had gestational hypertension. No uterine anomalies were recorded, four women (5.2\%) who underwent ECV had previous abdominal surgeries and four others (5.2\%) had a previous cesarean delivery along with normal fetal condition. Although no immediate complications from the ECV were recorded, 22 (28.6\%) experienced failure of ECV, of whom, eight women (36.4\%) required emergency cesarean delivery and 14 women (63.6\%) had an elective cesarean delivery.

In the association between the prognostic parameters and the rate of successful ECV, the gravidity status was 11 for nulliparous with a percentage equals $23.9 \%$ and 35 (76.1\%) for multiparous. Women with BMI between 25 to 29.9 had the highest successful rate of ECV (53.1\%). Maternal age older than 30 years old rated $60.9 \%$ in comparison to women younger (39.1\%). $<37$ weeks of gestation was $30.4 \%$, >39 weeks was $13.0 \%$ and between 37 to 39.6 gestational weeks were the highest rate and accounted for $56.5 \%$. In relation to the placenta location, pregnant women with Posterior placenta were documented with successful rate of $55.6 \%$, followed by the anterior location $31.1 \%$. For fetal conditions, the success rate was more with an estimated fetal weight of more than 2500 (73.9\%).

\section{Discussion}

This present study illustrated that the outcome of ECV and the final mode of delivery is statistically significant $(\mathrm{P}=0.000)$ as $\mathrm{ECV}$ procedure documented a success rate in $59.6 \%$ of women, and the overall vaginal delivery rate after a successful ECV was $87 \%$ and the rate for both elective and emergency cesarean section was $13 \%$. These findings are consistent with a study that was reported in Taif, Saudi Arabia that showed $84.1 \%$ of the women delivered vaginally and $14.5 \%$ of them underwent cesarean section after a successful ECV. It is suggested that ECV should be offered to all women with a breech presentation to minimize the danger of non-cephalic presentation at term and decrease the risk of cesarean section [13].

In our study, we found that ECV success significantly increases with maternal age older than 30. In contrast, ECV success rate decreases as maternal age become $<30(\mathrm{P}=0.028)$ although there could be other prognostic parameters that are affecting the success rate of ECV along with the maternal age such as BMI which was classified according to WHO classification and due to the expectation of a mean weight rise of $9 \mathrm{~kg}$ during pregnancy, we did our calculation by subtracting the expected mean 9-kg weight rise based on Burgos et al [14]. And as BMI was in the normal range and the gravidity status was multigravida along with $>30$ years of maternal age the success rate increases from $37.5 \%$ to $54.3 \%$. The rate of success is proportionally related 
to both EFW and age. Pregnant women older than 30 years old and at 37-39.6 weeks of gestation their rate of successful ECV increases (58.3\%).

Numerous studies have investigated the variables affecting ECV's success rate; however, the majority of research varies significantly in terms of methodological quality and sample size. As well as maternal weight, BMI, amniotic fluid index, and estimated fetal weight were the factors most frequently used in previous studies. Both Kok et al. and Burgos et al. found that parity and AFI are predictive for successful ECV procedure. [14,15] Which in fact supports our result that reports a higher success rate $(76.1 \%)$ for multiparous women in comparison to nulliparous (23.9\%). However, all our data for AFI were within the normal range which is between 7 and 25 centimeters, these two previous studies suggested that AFI below 10 centimeters had an impact on the outcome of the ECV and therefore this may lower the success rate. $[2,16,17]$ Furthermore, Shalev et al analyzed many variables and found a significant association between successful version and EFW [18] as our study showed EFW is related to the outcome of ECV and optimum EFW for a successful ECV was $2686 \mathrm{~g}$.

BMI was also taken under consideration in our study and so many other studies $[2,14,16,19]$, as they reported that women with normal BMI are more likely to have a successful ECV than women with greater BMI.

\section{Conclusions}

In conclusion, successful ECV cases has recorded $59.6 \%$ of women. The results from this study demonstrated vaginal delivery rate after a successful ECV was $87 \%$, While the rate of emergency and elective cesarean section was $13 \%$.There is significant increase in the incidence of spontaneous vaginal delivery after ECV. Outcome of ECV is affected by many prognostic parameters such as: parity, maternal age (women older than 30 years old), 37-39.6 weeks of gestation, BMI within normal range, AFI within normal range and the optimal EFW $2686 \mathrm{~g}$. It is suggested that ECV should be offered to all women with a breech presentation to minimize the danger of non-cephalic presentation at term and decrease the risk of caesarean section.

\section{Additional Information \\ Disclosures}

Human subjects: Consent was obtained or waived by all participants in this study. Institutional review board (IRB) King Abdulaziz University Hospital issued approval NA. Animal subjects: All authors have confirmed that this study did not involve animal subjects or tissue. Conflicts of interest: In compliance with the ICMJE uniform disclosure form, all authors declare the following: Payment/services info: All authors have declared that no financial support was received from any organization for the submitted work. Financial relationships: All authors have declared that they have no financial relationships at present or within the previous three years with any organizations that might have an interest in the submitted work. Other relationships: All authors have declared that there are no other relationships or activities that could appear to have influenced the submitted work.

\section{References}

1. Melo P, Georgiou EX, Hedditch A, Ellaway P, Impey L: External cephalic version at term: a cohort study of 18 years' experience. BJOG. 2019, 126:493-9. 10.1111/1471-0528.15475

2. Isakov O, Reicher L, Lavie A, Yogev Y, Maslovitz S: Prediction of success in external cephalic version for breech presentation at term. Obstet Gynecol. 20191, 133:857-66. 10.1097/AOG.0000000000003196

3. Lau TK, Lo KW, Wan D, Rogers MS: Predictors of successful external cephalic version at term: a prospective study. Br J Obstet Gynaecol. 1997, 104:798-802. 10.1111/j.1471-0528.1997.tb12023.x

4. Hofmeyr GJ, Kulier R: Cephalic version by postural management for breech presentation . Cochrane Database Syst Rev. 2012, 10:CD000051. 10.1002/14651858.CD000051.pub2

5. Grootscholten K, Kok M, Oei SG, Mol BW, van der Post JA: External cephalic version-related risks: a metaanalysis. Obstet Gynecol. 2008, 112:1143-51. 10.1097/AOG.0b013e31818b4ade

6. Mezei G, Jurus D, Ural S: P16. 07: Prognostic parameters for ultrasound-guided external cephalic version: a retrospective review of 92 cases. Ultrasound Obstet Gynecol. 20111, 38:226. 10.1002/uog.9823

7. Chaudhary S, Contag S, Yao R: The impact of maternal body mass index on external cephalic version success. J Matern Fetal Neonatal Med. 2019, 32:2159-65. 10.1080/14767058.2018.1427721

8. de Hundt M, Velzel J, de Groot CJ, Mol BW, Kok M: Mode of delivery after successful external cephalic version: a systematic review and meta-analysis. Obstet Gynecol. 2014, 123:1327-34. 10.1097/AOG.0000000000000295

9. Kok M, Van Der Steeg JW, Mol BW, Opmeer B, Van Der Post JA: Which factors play a role in clinical decision-making in external cephalic version?. Acta Obstet Gynecol Scand. 2008, 87:31-5. 10.1080/00016340701728075

10. Lavie A, Reicher L, Avraham S, Ram M, Maslovitz S: Success rates of early versus late initiation of external cephalic version. Int J Gynaecol Obstet. 2019, 145:116-21. 10.1002/iigo.12774

11. Thissen D, Swinkels P, Dullemond RC, van der Steeg JW: Introduction of a dedicated team increases the success rate of external cephalic version: a prospective cohort study. Eur J Obstet Gynecol Reprod Biol. 2019, 236:193-7. 10.1016/j.ejogrb.2019.04.002

12. Levin G, Rottenstreich A, Weill Y, Pollack RN: External cephalic version at term: a 6-year single-operator 


\section{Cureus}

experience. Birth. 2019, 46:616-22. 10.1111/birt.12429

13. Kew N, DuPlessis J, La Paglia D, Williams K: Predictors of cephalic vaginal delivery following external cephalic version: an eight-year single-centre study of 447 cases. Obstet Gynecol Int. 2017, 2017:3028398. $10.1155 / 2017 / 3028398$

14. Burgos J, Melchor JC, Pijoán JI, Cobos P, Fernández-Llebrez L, Martínez-Astorquiza T: A prospective study of the factors associated with the success rate of external cephalic version for breech presentation at term. Int J Gynaecol Obstet. 2011, 112:48-51. 10.1016/j.ijgo.2010.07.023

15. Kok M, van der Steeg JW, van der Post JA, Mol BW: Prediction of success of external cephalic version after 36 weeks. Am J Perinatol. 2011, 28:103-10. 10.1055/s-0030-1262909

16. Ben-Meir A, Erez Y, Sela HY, Shveiky D, Tsafrir A, Ezra Y: Prognostic parameters for successful external cephalic version. J Matern Fetal Neonatal Med. 2008, 21:660-2. 10.1080/14767050802244938

17. Tasnim N, Mahmud G, Khurshid M: External cephalic version with salbutamol - success rate and predictors of success. J Coll Physicians Surg Pak. 2009, 19:91-4.

18. Shalev E, Battino S, Giladi Y, Edelstein S: External cephalic version at term--using tocolysis . Acta Obstet Gynecol Scand. 1993, 72:455-7. 10.3109/00016349309021134

19. Mowat A, Gardener G: Predictors of successful external cephalic version in an Australian maternity hospital . Aust N Z J Obstet Gynaecol. 2014, 54:59-63. 10.1111/ajo.12152 